\title{
La comunicación en hospitales: enfoques y perspectivas
}

Communication in hospitals: approaches and perspectives

\author{
María Teresa Poccioni tpoccioni@gmail.com
}

http://orcid.org/0000-0002-6941-9665

Facultad de Periodismo y Comunicación Social; Universidad Nacional de La Plata/ Universidad Arturo Jauretche (Argentina)

\section{Resumen}

En este artículo se presenta el estado del arte en torno a comunicación en organizaciones hospitalarias, dando cuenta de las investigaciones y trabajos más recientes que abordan esta temática desde distintos aspectos tales como la comunicación interna y externa, la relación Question, Vol. 1, N. ${ }^{\circ}$ 63, julio-septiembre 2019. ISSN 1669-6581 
entre comunicación y calidad de atención, entre otros. A partir de los trabajos revisados se establecen ejes de análisis en esta línea de investigación, así como también se reflexiona en torno a las perspectivas teóricas vigentes y la necesidad de discutir nuevas formas de abordaje que incluyan las discusiones actuales en torno a comunicación organizacional.

Palabras clave: Comunicación; organización; hospitales.

\section{Abstract}

This article presents the state of the art around communication in hospital organizations. It shows the most recent research and articles related to this topic from its different aspects such as internal and external communication, the relationship between communication and quality of care, among others. Based on the reviewed works, the article offers analysis axes to be analysed in this line of research, as well as studies the current theoretical perspectives and the need to discuss new ways of approach that include current discussions around organizational communication.

Keywords: Communication; organization; hospitals.

En los últimos años hemos asistido en Argentina a una cada vez mayor consideración de la importancia de la comunicación en las organizaciones de salud, específicamente en instituciones hospitalarias. El Hospital de Alta Complejidad Juan Domingo Perón, de Formosa, inaugurado en el año 2003, el Hospital de Alta Complejidad en Red, Néstor Carlos Kirchner, de Florencio Varela (provincia de Buenos Aires) inaugurado en 2007, el Hospital Regional de Cañuelas (Cuenca Alta) que comenzó a funcionar en 2017, son algunos ejemplos de hospitales que en su origen contaron con un área específica de Comunicación. El Hospital Garrahan, de la ciudad de Buenos Aires, creado en 1987, si bien no contaba con un área específica de Comunicación, tuvo desde sus inicios una relación con los medios masivos muy fluida, y a partir de 2013 aproximadamente comienza a considerarse la necesidad de contar con un área de Comunicación.

Por otra parte, en distintas instituciones hospitalarias, si bien no cuentan con áreas específicas, en distintos momentos y frente a distintas circunstancias han ido incorporando profesionales de la comunicación o referentes a cargo de las tareas consideradas como pertenecientes al campo comunicacional, con distintas lógicas y dinámicas, planteándonos a quienes nos 
interesamos por este campo el desafío de analizar cómo se han ido configurando estos procesos, a qué objetivos responden, qué lugar ocupan en la gestión hospitalaria y qué resultados se esperan de la puesta en juego de estrategias comunicacionales hacia adentro y hacia afuera de la institución. Esto requiere un abordaje teórico que a partir de revisar las teorías clásicas acerca de la comunicación organizacional, tienda a adoptar una mirada vinculada a la complejidad de las organizaciones y a la comunicación desde una perspectiva estratégica.

En este artículo presentaremos una revisión del estado del arte en torno a comunicación y organizaciones de salud a partir de las publicaciones académicas realizadas en los últimos años.

Para esto, se llevó a cabo una búsqueda de artículos desde el año 2000 en adelante, publicados en América del Sur y España, en distintos repositorios y bibliotecas electrónicas, tales como SEDICI (repositorio de la Universidad Nacional de La Plata), Scielo (Biblioteca Electrónica Científica en Línea), BVS (Biblioteca Virtual de Salud), ResearchGate, entre otros sitios.

Entre los criterios utilizados, además del temporal y de ubicación geográfica, se tuvo en cuenta que se tratara de investigaciones de corte empírico, indagaciones concretas o artículos que dieran cuenta de líneas de investigación en el campo.

Los trabajos relevados giran en torno a distintas dimensiones tales como la comunicación interna (al interior de los equipos, entre directivos y trabajadores - profesionales y no profesionales-; en la relación médico-paciente o profesional de la salud-paciente; como herramienta de gestión); la gestión de la comunicación mediante la creación de áreas específicas; la comunicación externa en las organizaciones de salud (imagen del hospital; difusión de servicios que se brindan); la relación entre comunicación y calidad de atención en general; la comunicación médico-paciente y la comunicación de malas noticias; entre otros aspectos. Por otra parte, es necesario aclarar que en muchos de estos trabajos se trata de instituciones con realidades diferentes a las de los hospitales públicos argentinos, aunque las cuestiones conceptuales y metodológicas pueden ser revisadas y utilizadas como marco para pensar la comunicación en todo tipo de organizaciones de salud.

Los trabajos destinados a indagar en torno a los procesos de comunicación que se producen al interior de las organizaciones sanitarias, y el impacto que estos procesos tienen en la atención de los pacientes/usuarios de dichas organizaciones son escasos, si bien existe una tendencia creciente hacia la consideración de esta cuestión. En los artículos relevados, en algunos casos se trata de investigaciones concretas y en otros son propuestas de indagaciones teóricas en 
torno al tema, encontrando en algunas oportunidades que se abordan las dos formas de comunicación, interna y externa, en un mismo trabajo.

\section{Comunicación en la gestión hospitalaria}

Una de las cuestiones que surge como materia de reflexión e indagación es la necesidad de incorporar la dimensión comunicacional en la gestión hospitalaria, valorándola ya sea como una herramienta o como un "valor agregado" que permite optimizar los objetivos de las organizaciones de salud y lograr una mejor calidad de atención.

En este sentido, se considera que los directivos de las organizaciones hospitalarias deberían integrarla debido a "la necesidad de un perfil del directivo del futuro donde la comunicación sea uno de los valores más importantes que tiene que tener en cuenta para la mejora de la gestión. Parece evidente que la optimización de la gestión empresarial requiere que desde la dirección se sintonice con el profesional, y esto sólo es posible si se hace uso de una adecuada comunicación" (March, Prieto, Danet, Pérez y Martínez, 2009: 166). Esta mirada está en línea con una perspectiva empresarial, que es la que predomina en gran parte de los trabajos que circulan en torno a la comunicación organizacional.

En el artículo mencionado los autores presentan una investigación cuyo objetivo era conocer la percepción de los directivos de los hospitales españoles sobre el desarrollo de la comunicación interna en sus respectivas organizaciones. Dicho estudio se realizó a través de un cuestionario autoadministrado, de respuestas con escala de tipo Likert y respuestas múltiples, enviado por correo electrónico a directivos de hospitales españoles, y de la totalidad de los mismos se logró obtener 135 entrevistas con dicha modalidad. Más allá de las consideraciones que se pudiera hacer en torno a la metodología utilizada para la recolección de la opinión de los directivos en torno a la comunicación, es interesante que como punto de partida se haya tenido en cuenta la percepción de los responsables de la organización hospitalaria, ya que la gestión está teñida fundamentalmente de las características de sus gestores, además de las condiciones políticas, económicas y sociales que atraviesan toda institución de esta índole.

En este trabajo, se define a la comunicación como lo que permite lograr un lenguaje común en "la empresa" (u organización), con una orientación hacia la idea de transmisión de información en tanto su cometido es "contar a la organización lo que la propia organización está haciendo, es decir, transmite la cultura de la empresa, su misión, su visión, sus valores, sus mensajes, sus objetivos generales, sus principales noticias" (March et al., 2009: 167). Según los investigadores, de este modo se logra una mejor cohesión entre los trabajadores, lo cual 
redunda en eficacia y competitividad, propias de las empresas modernas. Además del enfoque empresarial, podríamos decir que predomina una concepción informacional de la comunicación, basada en la transmisión óptima de mensajes: como conclusión de su trabajo de investigación, los autores resaltan la importancia "de desarrollar canales formales bien establecidos en los planes de comunicación para que la información llegue a los públicos definidos y lo haga de la forma más clara posible" (Ibídem: 165).

Entre los trabajos que destacan el valor estratégico de la comunicación interna en los hospitales, se encuentran los producidos por Medina Aguerrebere (2012) y Medina Aguerrebere y Del Río Pérez (2015), quienes se preguntan si la comunicación interna hospitalaria puede ser considerada una herramienta estratégica de gestión, y buscan la respuesta analizando el contexto hospitalario, el rol de la comunicación institucional en los hospitales, y el carácter estratégico de la comunicación interna.

En relación al contexto hospitalario, Medina Aguerrebere (2012) establece que los hospitales son instituciones con una misión social, la cual es fundamentalmente mejorar la salud de las personas, y su característica principal es la de ser sistemas complejos formados a su vez por distintos subsistemas que deben articularse entre sí para lograr cumplir con su misión. A su vez estos subsistemas están conformados por personas, provenientes de distintas profesiones y contextos experienciales y a su vez, su tarea se desarrolla en presencia de quienes acuden a resolver su problema de salud, los "pacientes", lo cual según el autor genera dificultades para la gestión de los procesos de atención.

Es justamente en esos procesos, tanto en la interrelación entre los subsistemas o servicios del hospital, como en la relación con los pacientes, que el autor hace hincapié en la importancia de la comunicación, tanto interna como externa, como una dimensión donde se ponen en juego las distintas concepciones, experiencias y características de los actores de dichos procesos; podría decirse, como espacio de mediación de las prácticas y de los sentidos que las mismas generan.

Con respecto al rol de la comunicación institucional, al igual que March et al. (2009) parte de una concepción corporativa, que se adecua mejor a los hospitales privados. La vincula con la idea de "marca" o identidad, abarcando bajo ese nombre los valores, misión, visión, cultura e imagen de la institución. "La organización hospitalaria puede y debe apostar por el desarrollo de una arquitectura de marca que siente las bases de su política de comunicación institucional" (Medina Aguerrebere, 2012: 23). Sin embargo es consciente de las dificultades propias del ámbito hospitalario, aunque insiste en la necesidad de imponer de algún modo a la comunicación institucional interna y externa como actividad estratégica, ya que la misma genera desde su perspectiva varios beneficios para la organización, uno de los cuales 
constituye la satisfacción de los propios pacientes. Plantea que la comunicación como proceso relacional debe pensarse más allá del mero intercambio de información, en tanto "influye positivamente en el funcionamiento de las instituciones" (Ídem). Aquí podemos ver una conceptualización más amplia de la comunicación, no sólo como transmisión de información sino también como proceso relacional que influye directamente en los procesos, más en línea con una perspectiva sistémica de la comunicación.

El autor identifica lo que denomina "intangibles" del proceso hospitalario, refiriéndose de este modo a las características sociales, culturales, emocionales que atraviesan tanto el acto médico como la atención hospitalaria en general. Afirma que dichos intangibles, como así también la gran cantidad de personas que intervienen en la actividad hospitalaria y su diversidad en cuanto a roles y funciones, y por ende la necesidad de una buena coordinación entre ellos, son algunas de las razones para considerar a la comunicación interna como fundamental para el hospital, dentro del campo de la comunicación institucional.

Toma la siguiente definición de comunicación institucional de La Porte:

\begin{abstract}
Tipo de comunicación que favorece o promueve relaciones eficientes entre las personas que configuran los públicos internos de una organización, y que genera la confianza necesaria para coordinar adecuada y responsablemente todos los recursos disponibles en la consecución de la misión compartida. Su objetivo es mejorar la calidad del trabajo de la organización y llevar a cabo con mayor perfección la realización de su misión (Medina Aguerrebere, 2012: 24).
\end{abstract}

La comunicación adquiere valor estratégico en el ámbito hospitalario porque influye positivamente en empleados, pacientes y la institución en su conjunto. Cuando describe en qué aspectos se daría esa influencia positiva, se refiere a la información que circula al interior del hospital, necesaria para poder llevar adelante la tarea y para generar condiciones favorables de trabajo. A su vez, la información se refiere a varios temas: lo que llama la "arquitectura de la marca" o identidad, las estrategias y objetivos, las opciones de capacitación para el personal. Al existir un flujo informativo de estas características, según el autor, se mejora el trabajo en equipo y la coordinación de tareas, bajando así el nivel de estrés y generando mayor confianza entre los trabajadores. Es decir que para Medina Aguerrebere, la información crea lazos sociales, lo cual es importante fundamentalmente en situaciones de cambio o crisis en la institución, ya que la comunicación interna podría ayudar a empleados y a la organización a adaptarse a las nuevas situaciones. "La verdadera utilidad de la comunicación interna reside en 
su capacidad para ayudar a los empleados a convertir la estrategia en acción" (Medina Aguerrebere, 2012: 25).

Asimismo, el autor afirma que la comunicación interna influye positivamente en el funcionamiento organizacional del hospital, sobre todo en relación a la gestión de procesos internos, la optimización de los costos y la atención al paciente.

El autor concluye entonces que la comunicación interna debe considerarse una herramienta de gestión, ya que persigue distintos objetivos, recurre a diferentes estrategias y soportes de comunicación, influye en varios aspectos de la organización y concierne a todos los empleados, de allí que requiera de una gestión estratégica desde la dirección para orientar dicha actividad a la consecución de las metas del hospital. Además, es una herramienta estratégica de dirección porque muchas de las iniciativas que se llevan a cabo influyen en la calidad del servicio que se ofrece a los pacientes y por lo tanto, a la satisfacción del paciente y la marca de la institución. Finalmente sostiene que debe haber un departamento de comunicación interna que "asuma esta actividad de una forma autónoma y protocolizada (creación de protocolos y de un plan oficial de comunicación interna) en la que la colaboración con los demás departamentos de la institución, por un lado, y la formación del profesional de la salud en el ámbito de la comunicación interna, por otro, constituyan sus dos principales áreas de actividad" (Medina Aguerrebere, 2012: 26). Más adelante nos referiremos a los trabajos identificados en torno a esta idea.

\section{Comunicación al interior de los equipos de salud}

Otra forma de referirse a la comunicación interna en las organizaciones de salud es a través del análisis de lo que sucede al interior de los equipos de salud, sean de una misma profesión (entre médicos/as, enfermeros/as) o bien cumplan diferentes roles y funciones. En esta línea, es interesante el cruce que se realiza en algunos trabajos entre comunicación y seguridad del paciente, siendo este último un tema de mucha actualidad en el campo de los estudios sobre calidad de atención. Una de las bases de los desarrollos en torno a seguridad del paciente es el aforismo hipocrático Primum non nocere (definido como "antes que todo no dañar"), y que apuntaba a que el médico no estaba obligado a curar a su paciente pero sí a no dañarlo, o perjudicarlo en el proceso de atención. Actualmente existe una percepción extendida de que la comunicación en el equipo de salud es uno de los factores que influye en la generación de errores o "eventos adversos" en el proceso de atención, y que pueden tener consecuencias terribles para la vida del paciente. De allí que comience a ponerse en foco este tema, al igual 
que hace unos años se hizo en el ámbito industrial para evitar accidentes de trabajo. Con esa misma lógica, existen algunos trabajos orientados a reconocer que una "apropiada comunicación" es "uno de los factores centrales que sustentan la seguridad y calidad del trabajo en equipos que desempeñan actividades complejas" (Ceriani Cernadas, 2014: 114). Sin embargo lo que parece predominar en los equipos médicos es más bien una "comunicación ineficiente entre los profesionales y con el personal administrativo" (Ídem), lo que no permite mejorar la seguridad del paciente sino más bien perjudicarlo. Según este autor, una revisión de eventos que han provocado muerte o daño grave, denominados eventos centinela, (más de 3500) realizados por la Joint Commission de Estados Unidos "determinó que el $60 \%$ de las muertes y $50 \%$ de los daños graves se debieron a un fracaso en la comunicación del trabajo en equipo" (Ídem).

Por otra parte, existen trabajos que se interesan en analizar qué sucede en la comunicación entre los profesionales de salud en un momento específico de la tarea hospitalaria como es el "pase de guardia". Este es un tema que comienza a surgir mayoritariamente en el área de enfermería, ya que en el campo médico no suele existir bibliografía al respecto.

El objetivo que orienta estos estudios está vinculado al que señalábamos en relación a seguridad del paciente, de evitar complicaciones innecesarias que redunden en un perjuicio para el paciente. Un ejemplo es el trabajo realizado en el Comité de Investigación en Educación Médica del Departamento de Docencia e Investigación del Hospital Italiano de Buenos Aires, de alto prestigio en nuestro país. Eymann y otros autores (2014) llevan a cabo un estudio de percepción de la calidad de la comunicación en el pase de guardia en las áreas de cuidados intensivos de dicho hospital, observando la información sobre los pacientes que reciben los médicos al realizar el pase y la posibilidad de confundir la información de un paciente con la de otro. Si bien no expondremos aquí los resultados, nos interesa señalar que en este trabajo se entiende el pase de guardia como un "proceso interactivo de comunicación de datos específicos del paciente y de transferencia de responsabilidades con el propósito de mantener la continuidad de la atención en forma segura" (Belziti, Eymann, Durante, Pizarro, Carrió y Figari, 2014: 119). En la medida que se omitan o distorsionen datos en ese proceso de comunicación, pueden llegar a producirse errores médicos y repercutir en los cuidados que debe recibir el paciente. Un aspecto interesante de este estudio es que se busca identificar cuáles son las causas que conducen a que se generen dificultades en el intercambio de información en el pase de guardia, para poder intervenir y realizar mejoras.

$\mathrm{Si}$ bien en este caso se refiere fundamentalmente a la comunicación entre médicos, como hemos señalado esta preocupación aparece más fuertemente entre los enfermeros/as de los hospitales. Dan cuenta de ello estudios como los de Rivero Rosales y otros (2016); Guevara 
Lozano y Arroyo Marles (2015), donde se insiste en la idea de "fallas" en la comunicación en el pase de guardia e impacto negativo para la seguridad del paciente.

Otra mirada sobre la comunicación en los equipos de salud hace foco en la eficacia para el cumplimiento de las tareas, como por ejemplo el trabajo de Alonso y otros (2011). Este estudio se concentra en la comparación entre el clima emocional, la calidad de la comunicación, la valoración de responsables y dirección, la relación entre compañeros y los indicadores de resultados obtenidos en dos servicios tradicionales y una unidad de gestión clínica.

Los investigadores concluyen que la modalidad organizativa de unidad de gestión clínica favorece una mejor comunicación interna ya que el contexto hospitalario es un espacio donde se producen interacciones y relaciones dinámicas muy complejas que pueden generar "egoísmo y competitividad". En la Unidad de Gestión Clínica las relaciones resultaban ser comparativamente mejores y caracterizadas por darse en forma óptima entre mujeres, de allí que concluyen que "un modelo organizativo basado en la participación activa de los profesionales, en una comunicación positiva y en el compromiso profesional lleva a mejores resultados de trabajo y al establecimiento de relaciones interpersonales más positivas" (Alonso, Rubio, March y Danet, 2011). También señalan el lugar central que ocupan en estas prácticas los responsables de estas áreas y marcan como una cuestión importante a tener en cuenta que las características de la organización condicionan el modo de comunicación al interior de los equipos hospitalarios, siendo necesario indagar sobre dichas características y sobre dichos procesos de comunicación a la hora de diseñar medidas de mejora para el hospital.

\section{Comunicación con los pacientes y calidad de atención}

Si bien no vamos a profundizar en esta línea de trabajo, por demás interesante, que es la comunicación médico/paciente, o entre profesionales de la salud/pacientes, hemos encontrado algunos trabajos que incluyen esta temática dentro de lo que es necesario estudiar al interior de los hospitales en relación a la comunicación. Se incluye esta dimensión para identificar indicadores de satisfacción de los usuarios o pacientes de los servicios de salud, indicando que a mejor calidad de comunicación/información, mayor satisfacción por parte de los usuarios. Existen estudios que toman situaciones particulares, como el estudio de la comunicación que reciben las mujeres primíparas en un servicio de salud (Díaz-Sáez et al., 2011); con pacientes oncológicos (Rennó y Campos, 2014; Domínguez Nogueira et al., 2007) así como artículos que analizan a la comunicación interpersonal como factor de satisfacción (Dierssen Sotos et al., 2009; Dios Guerra et al., 2013) y el counseling o consejería como herramienta para mejorar la 
comunicación con el paciente (Martí-Gil et al., 2013). En general estos trabajos se enmarcan en el campo de estudios sobre calidad de atención en salud, e incluso pueden vincularse a otra línea de investigación en torno a lo que se denomina "humanización" de la atención (Gonçalves de Oliveira et al., 2006). Se trata de trabajos que incluyen a la comunicación como un indicador para medir la calidad de las organizaciones de salud.

\section{Sobre la creación de áreas de comunicación en hospitales}

En línea con lo planteado por Medina Aguerrebere en relación al valor estratégico de la comunicación interna para la gestión hospitalaria, varios trabajos remiten al análisis y propuestas en torno a la creación de áreas específicas, o departamentos, o lo que en España se conoce como Gabinetes de Comunicación en los hospitales. En ese sentido, Costa Sánchez analiza dichos departamentos o gabinetes en los hospitales públicos de Galicia asumiendo la importancia de la comunicación como "herramienta imprescindible para conseguir una imagen positiva interna-externa y en relación con los usuarios" (2011: 175).

A través de un estudio cuali-cuantitativo, da cuenta del relevamiento de quince hospitales españoles encontrando que en seis de ellos existía un gabinete de comunicación. Dichos hospitales compartían características similares, ya que se trataba de complejos hospitalarios (es decir, que agrupan varios hospitales y especialidades, generalmente de alta complejidad), de más de 250 camas y con una gran cantidad de empleados.

Para Costa Sánchez, "la comunicación constituye el elemento que aporta valor añadido al servicio hospitalario, pues a través de la comunicación puede adaptarse el servicio (hospitalario) a la idiosincrasia y necesidades de cada paciente" (2011: 175). Y esto produce usuarios satisfechos o con una buena valoración de la calidad de atención recibida.

Para esta autora, al ser la comunicación parte de los servicios que se les brindan a los usuarios, es fundamental que exista un área que pueda gestionarla, tanto en relación a necesidades internas como externas. Esta área, denominada gabinete o departamento, "debe encargarse de "diseñar, planificar, ejecutar, evaluar y coordinar la estrategia comunicativa hacia adentro y hacia afuera- que desea llevarse a cabo."

Si bien en España desde hace unos veinte años cobró impulso la importancia de la comunicación hospitalaria y la necesidad de contar con un área de estas características, tanto en el ámbito público como en el privado, comparten con nuestras escasas áreas de comunicación en hospitales el problema de que las mismas quedan relegadas la mayoría de las veces a funciones vinculadas únicamente a los medios masivos de comunicación. 
En la misma línea, Medina Aguerrebere y Del Río Pérez (2015) avanzan en la propuesta de cómo deberían estar organizadas estas áreas o Departamentos de Comunicación Institucional, cuyas funciones abarcarían la comunicación interna, externa, las relaciones con los medios, la organización de eventos, entre otras. Entre todas ellas, destaca lo que denomina la definición de la arquitectura de marca del hospital, es decir, su identidad, valores, misión, visión, cultura e imagen. El modelo de organización en el que están pensando, obedece a la lógica de los hospitales privados, para los cuales la imagen es muy importante en tanto deben "captar" a sus pacientes, entendidos como "clientes", que tienen distintas opciones de servicios de salud y por lo tanto hay que convencerlo de que va a cubrir su expectativa en cuanto a atención de la salud. Parte de la idea de que

\begin{abstract}
cuando un cliente entra en contacto con una marca, éste juzga si la imagen que él tiene de la misma es coherente con la realidad que se vive en ella (Quirke, 2000). Por eso, el Departamento de Comunicación Institucional, antes de implantar ninguna iniciativa concreta de comunicación, define la arquitectura de marca para, de este modo, cohesionar dichas iniciativas e influir positivamente en las percepciones de los stakeholdersdel hospital: pacientes, periodistas, autoridades públicas, etc. (Medina Aguerrebere y Del Río Pérez, 2015).
\end{abstract}

\title{
Estrategias de comunicación externa en las organizaciones de salud
}

Los trabajos que giran en torno a las estrategias de comunicación externa suelen estar orientados por el marco conceptual del marketing o "mercadeo", sobre todo si tenemos en cuenta que se trata de hospitales privados que, como ya dijimos, necesitan proyectar hacia la población una imagen de calidad, eficiencia y eficacia en la búsqueda de resultados a los problemas de salud que allí se traten (Sanguino Galván y Arroyo Mena, 1999). Los trabajos relevados son en general tesis de grado y posgrado, o proyectos de investigación, en los cuales o bien se realiza una descripción de los medios y estrategias que utilizan los hospitales para difundir sus servicios (Pelitti, 2016) o bien se realizan propuestas de planes de comunicación en ese sentido. Existen incluso manuales de comunicación en algunos hospitales donde se detallan las actividades propuestas para cada uno de los públicos del hospital. 


\section{Comunicación de malas noticias}

Para finalizar, podemos identificar otro grupo de trabajos que si bien se relacionan con lo ya señalado sobre la comunicación entre profesionales de salud y pacientes en el ámbito hospitalario, tienen una característica particular y últimamente están cobrando mucho interés sobre todo en el sector médico, como es la comunicación de malas noticias (Sobrino López, 2008; García Díaz, 2006; Bascuñán, 2013).

\section{Consideraciones finales}

Esta rápida revisión de trabajos en torno a la comunicación en hospitales permite vislumbrar todo un campo de estudios con una gran potencialidad, tanto a nivel académico como a nivel de la gestión hospitalaria. Sin embargo, es necesario realizar un análisis profundo de las perspectivas y conceptualizaciones que dan cuenta, para ir construyendo marcos teóricos que trasciendan los modelos tradicionales que predominan en gran parte de las investigaciones en este campo.

Si bien en algunos casos se resalta el valor estratégico asignado a la comunicación en la gestión de las organizaciones hospitalarias, lo que predomina es una visión "informacional" de la comunicación, y desde una perspectiva fuertemente "utilitaria" o "herramental" que no toma en cuenta la característica de transversalidad de la comunicación en las distintas dimensiones de la gestión hospitalaria.

No se ignora el fuerte componente que tiene la comunicación institucional en relación a la necesidad de informar o dar cuenta de sus actos, y la necesidad de influir o modificar en alguna medida las representaciones vigentes de su público, interno y externo. Como plantean Riorda y Elizalde:

la comunicación le aporta a la estrategia en sentido general la posibilidad de influir, de afectar o activar a personas, a grupos de personas y a organizaciones. No es posible poner en funcionamiento, transformar una estrategia en algo real, si no se utilizan diferentes niveles y dispositivos de comunicación (2013).

Hace ya bastante tiempo, Bateson y Ruesch (1984) definían a la comunicación incluyendo en ella a "todos los procesos a través de los cuales la gente se influye mutuamente", es decir como un proceso permanente e integrado, multidimensional y complejo, como matriz donde 
encajan todas las actividades humanas. Sin embargo, definiciones tan generales y abarcativas como la señalada requieren ser reorientadas hacia procesos concretos, ya que se corre el riesgo de pensar que como todo es comunicación, o como todo es un "problema de comunicación" - afirmación habitual en las organizaciones de salud - y la comunicación es inherente a los seres humanos, el margen de acción queda librado a la subjetividad de los actores involucrados.

De allí que nos interesa profundizar en una perspectiva de la comunicación que entienda a los procesos comunicacionales en las organizaciones de salud como fenómenos complejos e insertos en procesos políticos, económicos y sociales con los cuales interactúan y sin los cuales es imposible comprenderlos.

\section{Bibliografía}

Alonso, E.; Rubio, A.; March, J.C. y Danet, A. (2011). Clima emocional y comunicación interna en una unidad de gestión clínica y dos servicios hospitalarios tradicionales. Rev Calid Asist, 26(5), pp. 281-284.

Bascuñán, M. L. (2013). Comunicación de malas noticias en salud. REV. MED. CLIN. CONDES, 24(4), pp. 685-693.

Bateson, G. y Ruesch, J. (1984). Comunicación. La matriz social de la Psiquiatría. Barcelona: Paidós.

Belziti, C.; Eymann, A.; Durante, E.; Pizarro, R.; Carrió, S. y Figari, M. (2014). Comunicación en el pase de guardia en las áreas de cuidados intensivos en un hospital universitario. Estudio transversal. Arch Argent Pediatr, 112(2), pp. 119-123.

Ceriani Cernadas, J. (2014). La comunicación en los equipos de atención médica: un desafío esencial para mejorar la seguridad del paciente. Arch Argent Pediatr, 112(2), pp. 114115.

Costa Sánchez, C. (2011). El gabinete de comunicación del hospital. Propuesta teórica y acercamiento a la realidad de los departamentos de comunicación de los hospitales públicos de Galicia. Doxa Comunicación, 14, pp. 175-197.

Díaz-Saez, J.; Catalán-Matamoros, D.; Fernández Martineza, M. M. y Granados Gamez, G. (2011). La comunicación y la satisfacción de las primíparas en un servicio público de salud. GacSanit, 25(6), pp. 483-489. 
Dierssen Sotos, T.; Rodríguez Cundin, P.; Robles García, M.; Brugos Llamazares, V. Gomez Acebol, I. y Llorcal, J. (2009). Factores que determinan la alta satisfacción del usuario con la asistencia hospitalaria. An. Sist. Sanit. Navar, 32(3), septiembre-diciembre.

Dios Guerra, C.; Alba Dios, M. A.; Ruiz Moral, R; Jiménez García, J.; Pérula de Torres, L. A. y Rodríguez Borrego M. A. (2013). Valoración de la satisfacción de usuarios de consulta de enfermería en centros de salud a partir de indicadores de calidad técnicos y de comunicación, Revista online Enfermería Global, 31.

Domínguez Nogueira, C.; Expósito Hernández, J.; Barranco Navarro, J. y Pérez Vicente, S. (2007). Dificultades en la comunicación con el paciente de cáncer y su familia: la perspectiva de los profesionales. Revista de Calidad Asistencial, 22(1), pp. 44-49.

Elizalde, L. y Riorda, M. (2013). Comunicación gubernamental 360. Buenos Aires: La Crujía Ediciones.

Ferreira Deslandes, S. y Gomes, R. (2007). La investigación cualitativa en los servicios de salud: notas teóricas. En Magalhaes Bosi, M. L. y Mercado, F. J. (coordinadores). Investigación cualitativa en los servicios de salud. Buenos Aires: Lugar Editorial.

García Díaz, F. (2006). Comunicando malas noticias en Medicina: recomendaciones para hacer de la necesidad virtud. Medicina Intensiva, 30(9), pp. 452-459. Recuperado de http://scielo.isciii.es/scielo.php?script=sci arttext\&pid=S0210$\underline{56912006000900006 \& l n g=e s \& t I n g=e s}$

Gonçalves de Oliveira, B. R.; Collet, N. y Silveira Viera, C. (2006). A humanização na assistência à saúde. Rev Latino-am Enfermagem, 14(2), março-abril. Recuperado de www.eerp.usp.br/rlae

Guevara Lozano, M.; Arroyo Marles, L. (2015). El cambio de turno: un eje central del cuidado de enfermería. Revista en Línea Enfermería Global, 37.

March, J. C.; Prieto, M. A.; Danet, A.; Pérez, O. y Martínez, F. (2009). Los directivos hospitalarios y la comunicación interna: una asignatura pendiente. Revista Adm Sanitaria, 7(I), pp. 165-182.

Martí-Gil, C.; Barreda-Hernández, D.; Marcos-Pérez, G. y Barreira-Hernández, D. (2013). Counseling: una herramienta para la mejora de la comunicación con el paciente. Farmacia Hospitalaria, 37(3), 236-239. Recuperado de https://dx.doi.org/10.7399/FH.2013.37.3.559

Massoni, S. (2004). Estrategias de comunicación: un modelo de abordaje de la dimensión comunicacional para el desarrollo sostenible entendido como cambio social conversacional. Revista Temas y Debates, 8, Facultad de Ciencia Política y RRII, Universidad Nacional de Rosario. 
Massoni, S. (2007a). Comunicación estratégica: experiencias, investigación y planificación en marcha. Rosario, Argentina: Universidad Nacional de Rosario-Agencia Nacional de Promoción Científica y Tecnológica.

Massoni, S. (2007b). Moebius, estrategia y comunicación. Revista Académica Estrategias, III(7). Recuperado de www.fisec-estrategias.com.ar

Maxwell, J. A. (1996). Qualitative Research Design. An Interactive Approach. Thousand Oaks, California: Sage Publications.

Medina Aguerrebere, P. (2012). El valor estratégico de la comunicación interna hospitalaria. Revista de Comunicación y Salud, 2(1), pp. 19-28.

Medina Aguerrebere, P. y Del Río Pérez, J. (2015). Comunicación interna hospitalaria: una aproximación desde la creatividad. Revista Mediterránea de Comunicación, 6(2), pp. 215-227. Recuperado de http://mediterranea-comunicacion.org/

Nogueira, M. S.; Mendes, I. A. C.; Trevizan, M. A.; Fávero, N. y Hayashida, M. (1999). Entrega de turno: análisis de factores en la comunicación. Horizonte de Enfermería, 10(1), pp.818.

Pelitti, P. (2016). Estrategias de comunicación interna y externa de los hospitales públicos bonaerenses de la Región Sanitaria XI. Revista Question, 1(49).

Rennó, C. S. N. y Gómez Campos, C. J. (2014). Comunicação interpessoal: valorização pelo paciente oncológico em uma unidade de alta complexidade em oncologia. REME-Rev Min Enferm, 18(1), pp. 106-15.

Rivero Rosales, A. M.; Pérez Castro y Vázquez, J. A. y Recio España, F. I. (2016). Características de la comunicación durante el enlace de turno de enfermería: una perspectiva rumbo a la calidad del cuidado. Revista Conamed, 21(3).

Sanguino Galván, R. y Arroyo Mena, A. (1999). Marketing hospitalario: el estado de la cuestión. En La gestión de la diversidad. XIII Congreso Nacional, IX Congreso Hispano-Francés, Logroño, La Rioja, 16, 17 y 18 de junio. Recuperado de https://dialnet.unirioja.es/servlet/articulo?codigo $=565068$

Sobrino López, A. (2008). Comunicación de malas noticias. Semin Fund Esp Reumatol, 9, pp. 111-122. 